\title{
Intravitreal injections of anti-VEGF agents during COVID- 19 pandemic: clinical audit from Tanta University Hospital
}

\author{
Magdy Moussa • Muhammed Sameh Elshorbagy • Ayman Emarah • \\ Raouf Gaber · Omar M. Moussa • Mohamed El-Bradey • Ahmed Roshdy Alagorie • \\ Osama A. Sorour $\mathbb{1 0}$
}

Received: 13 September 2020/ Accepted: 9 January 2021 / Published online: 21 January 2021

(C) The Author(s), under exclusive licence to Springer Nature B.V. part of Springer Nature 2021

\begin{abstract}
Background The aims of this study were to provide real-life data about the effect of COVID-19 pandemic on the practice of anti-VEGF injections and to evaluate the safety of the modifications in the injection protocol imposed during the ongoing pandemic on the anatomical and functional outcome of patients.

Methods All patients attending Tanta University hospital for receiving intravitreal anti-VEGF injections were screened. Patients who were previously deferred according to a modified protocol implemented in the hospital in response to the pandemic or who demonstrated deviation from it were included for further analysis.

Results During the audit period, 83 patients attending for anti-VEGF injections were screened, of whom 40 met the abovementioned criteria and were included for analysis. In the deferred subgroup (11 eyes), predeferral mean values of $\log$ MAR best corrected visual acuity (BCVA) and central retinal subfield thickness (CST) were $1 \pm 0.23$ and
\end{abstract}

M. Moussa · M. S. Elshorbagy · A. Emarah .

R. Gaber · M. El-Bradey · A. R. Alagorie .

O. A. Sorour $(\square)$

Ophthalmology Department, Faculty of Medicine, Tanta

University, Elgeish street, Tanta 31111, Egypt

e-mail: Osama.soror@med.tanta.edu.eg

O. M. Moussa

Ophthalmology Department, Faculty of Medicine, Kafr-

Elsheikh University, Kafr-Elsheikh, Egypt
$444.57 \pm 200.1 \mu \mathrm{m}$, respectively. There was no significant change when the patients returned for their deferred injections, with the mean BCVA and CST values being $0.8 \pm 0.22$ and $413.71 \pm 237.7 \mu \mathrm{m}$, respectively ( $p=0.27$ and $p=0.12$ ). Moreover, 29 patients encountered a disturbed injection schedule, particularly skipping their injection appointments due to infection fear as found in 18 patients.

Conclusion The COVID-19 pandemic has imposed pressing challenges in maintaining essential health care while ensuring the prevention of spread of infection. Although the modified injection protocol confirmed to be safe for patients, the pandemic caused deflection from the optimum practice in the form of successive skipping of appointments and delays in the processing of patient injection schedules.

Keywords COVID-19 - Pandemic - Anti-VEGF . Diabetic macular edema $\cdot$ Choroidal neovascularization $\cdot$ Injection protocol

$\begin{array}{ll}\text { Abbreviations } \\ \text { Anti- } & \text { Anti-vascular endothelial growth factor } \\ \text { VEGF } & \\ \text { BCVA } & \text { Best corrected visual acuity } \\ \text { BRVO } & \text { Branch retinal vein occlusions } \\ \text { CNV } & \text { Choroidal neovascularization } \\ \text { CRVO } & \text { Retinal vein occlusions } \\ \text { DR } & \text { Diabetic retinopathy } \\ \text { DME } & \text { Diabetic macular edema }\end{array}$


FA Fluorescein angiography

OCT Optical coherence tomography

\section{Background}

The COVID-19 pandemic has presented unprecedented challenges to the health care services worldwide. Maintaining the fine balance between the necessity of continuous provision of essential health care and the protection of the society, the health personnel, and the regular patients from the transmission of infection with the virus has become a complicated task. To achieve this aimed balance, diverse modifications of the standard treatment protocols were adopted during this pandemic [1].

Intravitreal injection of anti-VEGF drugs is the treatment of choice in several retinal diseases, including diabetic retinopathy (DR), diabetic macular edema (DME), choroidal neovascularization (CNV), and central and branch retinal vein occlusions (CRVO/ BRVO) with macular edema. Most of these patients are considered to be at high risk with regard to COVID-19 infection morbidity, as they are generally elderly and suffering from several underlying medical conditions, e.g., diabetes, hypertension, and cardiovascular diseases. Therefore, it is necessary to take utmost caution to protect this vulnerable group, besides not risking their vision due to nonjustifiable treatment delay [2]. In addition, anti-VEGF drug injections are routinely preceded by preinjection visits to the clinic; preinjection investigations by either optical coherence tomography (OCT), fluorescein angiography (FA), and/or optical coherence tomography angiography (OCTA); and postinjection followup clinic visits and investigations, which overblows the risk of contraction and transmission of infection [3]. As a result, several national and international recommendations were introduced to guide the optimum practice of anti-VEGF injections during this pandemic [4-6]. We implemented similar modifications to the routine anti-VEGF intravitreal injection protocol at the Ophthalmology Department, Tanta University Hospitals (OD-TUH), which is a governmental tertiary eye care facility in the middle of the Egyptian Nile Delta offering health care services to a population of more than 7 million people in this region.

Auditing of the anti-VEGF practice during the pandemic is of paramount importance to evaluate the impact of the applied injection protocol modifications and the influence of the ongoing pandemic on the injection service, particularly ahead of the anticipated second larger wave of COVID-19 infection in the coming fall.

\section{Materials and methods}

This clinical audit was approved by the ethical committee at the Faculty of Medicine, Tanta University, and was registered with code $=33883 / 6 / 20$. Informed consent was obtained from all recruited patients, and the study was conducted according to the regulations of the Declaration of Helsinki.

A partial lockdown was applied in Egypt beginning from mid-March 2020 till the end of June 2020. Several sectors from the society were instructed to stay home, including students and workforce with underlying chronic diseases such as diabetes. The remaining workforce in several economic and service sectors was reduced to $50 \%$ from the original to help social distancing and mitigate virus transmission. This protocol was also applied to the health care workers not working directly with COVID-19 patients. The OD-TUH has initiated the application of the following modified injection protocol (Table 1) and protective measures since mid-March.

Protective measures during the injection appointments

1. Before admission of the patients to the hospital, screening for fever or any respiratory symptoms suggestive of COVID-19 is checked. In case of any doubt about the patient's condition, he/she is instructed to return home and call the national number of COVID-19 services. Similarly, all health care personnel in the hospital are continuously monitored for signs or symptoms suggestive of infection.

2. Reduction in the number of patients in every injection setting is encouraged. Moreover, social 
Table 1 Modified protocol of anti-VEGF drugs injections during the covid-19 pandemic in OD-TUH

\footnotetext{
New patients

Injections were deferred in patients with DME and BRVO with good visual acuity (Snellen letters BCVA $\geq 6 / 12$ ), with follow-up scheduled after 3-4 months

Patients with DME and BRVO with worse BCVA $(<6 / 12)$ and confirmed wet AMD or CRVO patients, a loading 3-6 anti-VEGF injections were decided with 4-6 weeks interval between injections. No OCT imaging except after the end of the loading dose. Additionally, patients are instructed about the postinjection care and any warning signs, e.g., redness, pain, or diminished visual acuity, and the pre-pandemic routine postinjection visits are nulled

Panretinal photocoagulation (PRP) replaced anti-VEGF injections for proliferative diabetic retinopathy when possible

Returning patients (patients under review by the hospital retina service)

Patients on prior treat and extend (TEX) injection regimen with established extension period continued this interval period between injections

Patients on prior PRN regimen for long period ( $\geq 1$ year) with stable DR, BRVO/CRVO, and DME were deferred for 4-6 months and for 3-4 months in CNV/PCV patients. Instructions about vision self-screening are given to patients, with emphasis on hospital calls in case of any deterioration

Patients just completing their loading dose or on recent PRN injection regiment were deferred their injections for 3-4 months in DME and CRVO/BRVO with macular edema, and for 2-3 months in CNV/PCV patients, with new OCT at the first postdeferral appointment
}

distancing in the reception area, with at least $2 \mathrm{~m}$ between patients, is strictly applied.

3. Injections are performed in the operating theater (OT) under complete aseptic conditions.

4. Patients' wearing of masks, gown, and cap in the OT is mandatory. Health care personnel should wear the complete personal protective equipment (PPE).

5. Adequate interval between patients, sufficient draping of patients, and limitations of talks before and after injection are encouraged.

\section{Data collection}

Data were collected during the period from June 28 to July 7, 2020. All patients coming for anti-VEGF injections in the retina service at the OD-TUH during this period were subjected to initial screening. Primary screening included the indication of the anti-VEGF injection, the time when the decision of injection was taken to identify any delay, assessing whether the antiinfection maneuvers were fulfilled, and assessing whether the anti-VEGF practice adheres to the standards. Only the subset of patients who were deferred anti-VEGF injections according to the above-described protocol (Table $1 / 2 b, c)$ or in whom deviation of the injection practice from the protocol was included for further analysis.
Data sources from the latter subgroup of patients (audit cohort) included the complete history, checking of their medical records, and ophthalmological examination before their scheduled injection. The following data were collected: patients' demographic data consisting of age, sex, and any medical conditions. In addition to the indication of the anti-VEGF injection, the following details were collected: the time when the decision for the injection was made and the causes of any delay after this decision, history of previous anti-VEGF injections, and BCVA in logMAR and CST during the period of the study and at the final visit, if applicable.

Aim of the audit and outcome measures

- To evaluate the coincidence of the anti-VEGF injection practice with standard guidelines during the ongoing pandemic and identification of any deflection and its cause if present.

- Assessment of the effect of the modifications in the injection protocol on the functional and anatomical outcome of the patients.

Primary outcome measurements were the change in $\log$ MAR BCVA and CST values in the deferred patients according to the modified protocol and the percentage of patients with disturbed anti-VEGF injections due to COVID-19-related causes. 
Statistical analysis

Statistical analysis was conducted using SPSS version 25 (IBM Corp, NY, USA). Distribution of the variables was evaluated using the KolmogorovSmirnov test and the Shapiro-Wilk test. Parametric data were expressed in the form of mean and standard deviation, and nonparametric data were expressed in the form of percentages. The Wilcoxon test was used for analyzing the change in BCVA and CST measurements within the deferred group.

\section{Results}

Among 83 patients who received the anti-VEGF injection during the audit period, 40 patients (48.2\%) experienced delay or interruption from their previous injection schedule or were deferred the anti-VEGF injection according to the modified protocol (Fig. 1). The mean age of these patients was $57.65 \pm 12.8$ years; 17 patients were males $(42.5 \%)$ and 23 were females (57.5\%). Indications for the injection were diabetic macular edema in 29 eyes (72.5\%), CNV in 7 eyes (17.5\%), and CRVO/BRVO in 4 eyes $(10 \%)$. We found $100 \%$ adherence of the working staff to the established anti-infective measures. Till the date of the end of our audit, there was no confirmed case of COVID-19 among the working staff in the retina service at the OD-TUH.

We found that 12 eyes (30\%) had not received their scheduled $1^{\text {st }}$ anti-VEGF injection on time and were attending during the week of the study to receive this delayed $1^{\text {st }}$ injection. In this subgroup, 10 eyes were receiving anti-VEGF injections for DME, and 2 eyes were receiving them for $\mathrm{CNV}$. The mean duration of delay was $83.5 \pm 31$ days, for which the causes were skipping their appointment due to fear of infection with COVID-19, which was the primary cause in 7 patients $(58.3 \%)$, delay in the processing of their insurance paperwork in 3 patients $(25 \%)$, delay of patient appointment by the hospital due to administrative or logistic issues in 1 patient $(8.3 \%)$, and other causes in 1 patient (Fig. 2).

Similarly, 17 patients $(42.5 \%)$ encountered interruption in their scheduled loading of anti-VEGF dose after the 1st or 2nd injections. Among this group, 10 eyes were receiving treatment for DME (58.8\%), 4 eyes were receiving for CRVO/BRVO (23.5\%), and 3 eyes $(17.6 \%)$ were receiving it for CNV. The average duration of this interruption was $63 \pm 27$ days. Fear of infection with COVID-19 was the primary cause of this interruption in the injection schedule in 7 patients $(41.1 \%)$, delay in the processing of insurance paperwork was the cause in 4 patients $(23.5 \%), 4$ patients reported skipping of appointments due to their belief that one injection is sufficient to stabilize the retinal condition until the end of the pandemic, and finally hospital-related issues were the cause of this interruption in 2 patients (11.7\%) (Fig. 2).

In 11 patients, the injection was deferred according to the abovementioned protocol (Table $1 / 2 \mathrm{~b}, \mathrm{c}$ ) during the pandemic. Nine eyes (81.8\%) had DME, and two eyes were receiving injections for CNV (18.2\%). Patients previously received a mean of $4 \pm 1.1$ injections. The average time of their deferral was $88.7 \pm 14.8$ days. The mean $\log$ MAR BCVA before this deferral was $1 \pm 0.23$, which was found to be $0.8 \pm 0.22$ at the auditing time $(\mathrm{p}=0.27)$. Similarly, predeferral mean CST value was $444.57 \pm 200.1 \mu \mathrm{m}$, whereas at the time of audit, this value was $413.71 \pm 237.7 \mu \mathrm{m}(\mathrm{p}=0.12)$.

\section{Discussion}

The COVID-19 pandemic has led to the adoption of strict protective measures by several governments to control its spread. There was a partial/complete lockdown in several countries for variable time periods. In Egypt, there was a partial lockdown for more than 3 months, with significant downsizing of workforce, including health care personnel not directly involved in the care of COVID-19 patients.

The global fight against the COVID-19 virus appears to be a long battle. There is a prompt need for adjustments of several ophthalmological practices, as well as continuous reassessment of these modifications, to ensure the provision of best care to our patients and to protect them and ourselves from infection, meanwhile maintaining their vision.

In the OD-TUH, besides the application of the standard anti-infection and sanitation measures, we provided a modified protocol for anti-VEGF injections based on recommendations from the guidelines of various ophthalmic societies, which fits our patients and our community. In our modified protocol, we adhered to the least possible injection frequency and 


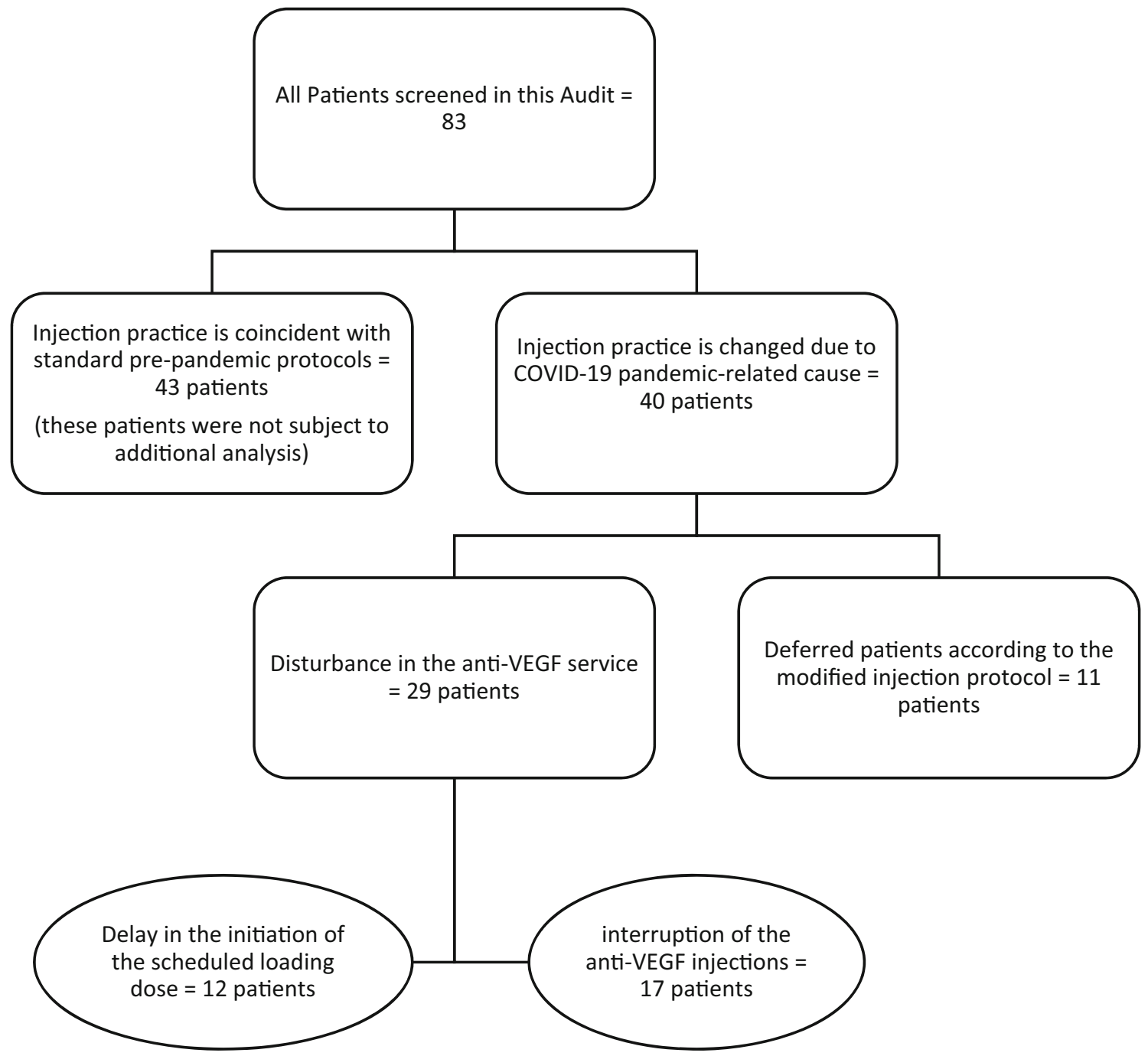

Fig. 1 Flowchart of the patients that are screened during this clinical audit

the longest possible interval between injections after the loading dose. In addition, we chose the short-term deferral of patients who are unlikely to suffer from vision deterioration during this short period. This deferral was applied for patients with DME and BRVO with good initial BCVA and for all patients after the loading dose, except in case of wellestablished injection intervals in patients we were following for long time before the pandemic. Our deferral of DME patients with good initial BCVA is comparable with the findings of the DRCR.net Protocol V study, which demonstrated no significant differences in visual outcome in these patients when they were treated with anti-VEGF or laser or were merely observed [7]. Conversely, a delay in treatment of CNV patients was mostly associated with a poorer visual outcome [8]. However, the FLUID study demonstrated that $\mathrm{CNV}$ patients who are treated with an extended protocol tolerated residual subretinal fluid and achieved a comparable final BCVA to that in patients in whom the treatment was aimed at resolving all the fluid [9]. These results support the possibility of treatment deferral in selected CNV patients.

The optimal treatment regimen after the initial loading dose remains highly controversial [10]. Unless the best interval was known for a patient based on previous visits, we extended the interval to the maximum by deferring patients for 2-6 months 


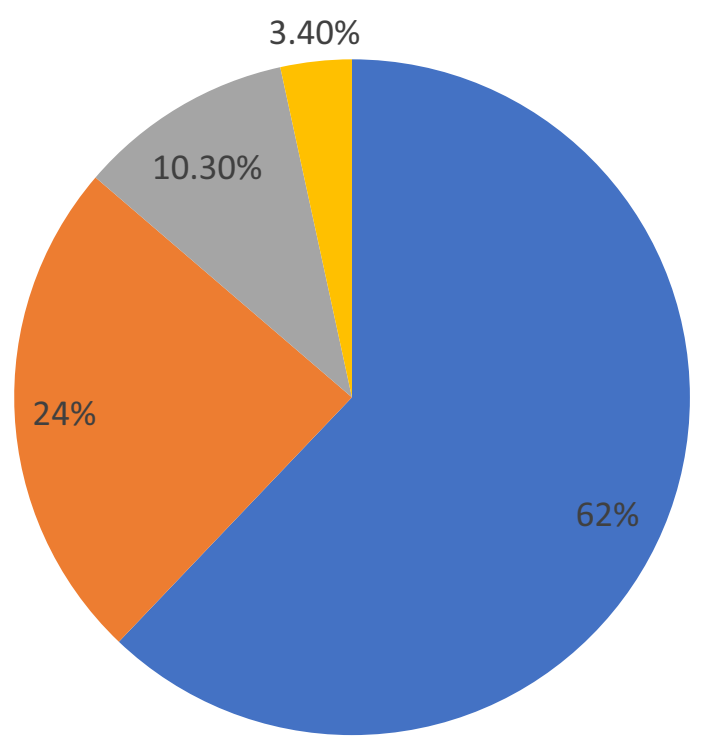

Fear of infection with COVID-19

Errors by hospital staff
Delay in processing of insurnace papers

other causes

Fig. 2 Causes of delay in initiation or interruption of the anti-VEGF injection schedule in the audited patients. a Pie chart illustrating the relative percentage of different causes of the disturbance in anti-VEGF practice during the covid-19 pandemic

according to their underlying retinal disease and clinical condition. During the pandemic, communication with patients via phone calls or messaging is extremely important to avoid delay in the provision of a needed care and for assurance of patients.

As a period of 3 months has elapsed from the application of these modifications, and the first clusters of deferred patients were returning for their appointments, it was necessary to assess the effect of this modified protocol on our patients and to evaluate the impact of the pandemic on the ongoing anti-VEGF injection practice in general.

The pandemic has a deep impact on patients requiring anti-VEGF injections. Approximately 25\% of patients screened in this audit have skipped their previous appointments due to their fear of contracting the infection, leading to either delay in the initiation of the scheduled anti-VEGF injection or interruption of the injections. Patients' fear demonstrates the important balance in handling the pandemic in the media. Besides the certain danger of COVID-19, other medical causes that may cause patient morbidity should not be overlooked. In addition, downsizing of workforce leads to some mistakes in patients' appointments or lagging of the processing of their insurance approvals, which causes a similar delay or interruption of the injection schedule.

Regarding the deferred patients according to the modified protocol, we did not find significant deterioration of mean vision nor the retinal edema in these patients, which emphasizes the safety of this protocol for our patients.

Our applied modified protocol has some similarities to the guidelines provided by the Royal College of Ophthalmologists [5], i.e., both deferred patients with low risk of vision loss or stable condition for 3-6 months. To the best of our knowledge, our study is the first in the literature to evaluate the effect of these modified protocols or to provide real-life data about the anti-VEGF practice during the COVID-19 pandemic. However, our audit has some limitations, including the small period of screening that led to the inclusion of a small number of patients. Moreover, the majority of our included patients had DME, with a small number of patients suffering from other retinal disorders, which prevented us from performing a 
subgroup analysis to evaluate the effect on each retinal lesion per se.

In conclusion, the modified anti-VEGF injection protocol adopted during this pandemic to minimize the risk of COVID-19 infection for both the patient and the health care personnel proved to be safe in maintaining the vision and clinical condition of patients. In addition, it allowed the allocation of the available limited resources for prioritizing treatment of patients at higher risk of irreversible vision loss. However, the pandemic had a profound effect on the anti-VEGF injection practice because a higher number of patients skipped their appointments or there was a delay in the processing of their schedule.

Acknowledgements Authors like to thank Muhammad Abouhamid, at Tanta university hospital, Tanta, Egypt, for his assistance during this study

Author contributions All authors contributed equally to the manuscript, and all authors read and approved the final version.

\section{Funding None.}

Data availability The data that support the findings of this study are available from Tanta University Hospital. Data are available from the corresponding author upon reasonable request and after permission of Tanta University Hospital.

\section{Compliance with ethical standards}

Conflict of interest The authors declare that they have no conflict of interests.

Ethical approval This clinical audit was approved by the ethical committee at the Faculty of Medicine, Tanta University, and was registered with code $=33883 / 6 / 20$.

\section{References}

1. Lai THT, Tang EWH, Chau SKY, Fung KSC, Li KKW (2020) Stepping up infection control measures in ophthalmology during the novel coronavirus outbreak: an experience from Hong Kong. Graefe's Arch Clinic and Exp Ophthalmol Albrecht von Graefes Archiv fur klinische und experimentelle Ophthalmologie 258:1049-1055
2. Agarwal D, Kumar A (2020) Managing intravitreal injections in adults in COVID-19 and post-COVID-19 era- Initial experiences. Indian J Ophthalmol 68:1216-1218

3. Romano MR, Montericcio A, Montalbano C, Raimondi R, Allegrini D, Ricciardelli G, Angi M, Pagano L, Romano V (2020) Facing COVID-19 in Ophthalmology department. Curr Eye Res 45:653-658

4. Shmueli O, Chowers I, Levy J (2020) Current safety preferences for intravitreal injection during COVID-19 pandemic. Eye 34:1165-1167

5. Korobelnik JF, Loewenstein A, Eldem B, Joussen AM, Koh A, Lambrou GN, Lanzetta P, Li X, Lovestam-Adrian M, Navarro R et al (2020) Guidance for anti-VEGF intravitreal injections during the COVID-19 pandemic. Graefe's Arch Clinic Exp Ophthalmol Albrecht von Graefes Archiv fur klinische und experimentelle Ophthalmologie 258:1149-1156

6. Sengupta S, Honavar SG, Sachdev MS, Sharma N, Kumar A, Ram J, Shetty R, Rao GS, Ramasamy K, Khanna R et al (2020) All India ophthalmological society - Indian journal of ophthalmology consensus statement on preferred practices during the COVID-19 pandemic. Indian J Ophthalmol 68:711-724

7. Baker CW, Glassman AR, Beaulieu WT, Antoszyk AN, Browning DJ, Chalam KV, Grover S, Jampol LM, Jhaveri CD, Melia M et al (2019) Effect of initial management With Aflibercept vs. Laser Photocoagulation vs. observation on vision loss among patients with diabetic macular edema involving the center of the macula and good visual acuity: a randomized clinical trial. JAMA 321:1880-1894

8. Lim JH, Wickremasinghe SS, Xie J, Chauhan DS, Baird PN, Robman LD, Hageman G, Guymer RH (2012) Delay to treatment and visual outcomes in patients treated with antivascular endothelial growth factor for age-related macular degeneration. Am J Ophthalmol 153:678-686

9. Guymer RH, Markey CM, McAllister IL, Gillies MC, Hunyor AP, Arnold JJ, Investigators F (2019) Tolerating subretinal fluid in neovascular age-related macular degeneration treated with Ranibizumab using a treat-and-extend regimen: FLUID study $24-$ month results. Ophthalmology 126:723-734

10. Lanzetta P, Loewenstein A (2017) Fundamental principles of an anti-VEGF treatment regimen: optimal application of intravitreal anti-vascular endothelial growth factor therapy of macular diseases. Graefe's Arch Clinic Exp Ophthalmol Albrecht von Graefes Archiv fur klinische und experimentelle Ophthalmologie 255:1259-1273

Publisher's Note Springer Nature remains neutral with regard to jurisdictional claims in published maps and institutional affiliations. 